\title{
Predicting adverse outcomes in end stage renal disease: machine learning applied to the United States Renal Data System
}

\author{
Zeid Khitan, MD', Alexis D. Jacob, MD², Courtney Balentine, MD², \\ Adam N. Jacob, BS ${ }^{3}$, Juan R. Sanabria, MD'1, Joseph I. Shapiro, MD'1
}

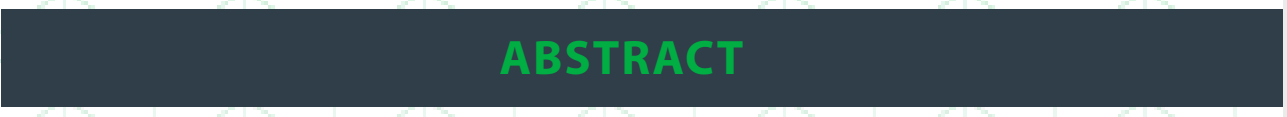

We examined machine learning methods to predict death within six months using data derived from the United States Renal Data System (USRDS). We specifically evaluated a generalized linear model, a support vector machine, a decision tree and a random forest evaluated within the context of K-10 fold validation using the CARET package available within the open source architecture R program. We compared these models with the feed forward neural network strategy that we previously reported on with this data set.
Author affiliations are listed at the end of this article.

Correspondence to: Joseph I. Shapiro, MD Marshall University Joan C. Edwards School of Medicine shapiroj@marshall.edu

\section{KEYWORDS}

hypertension, blood pressure, chronic renal disease, correlation, machine learning

\section{INTRODUCTION}

Patients with end stage renal disease (ESRD) have an extremely high extra renal morbidity and age adjusted mortality compared with the general population in the United States..$^{1-3}$ A number of factors have been identified which predict risk in this patient population, and some of these factors are reasonably powerful at predicting risk. ${ }^{4-8}$ We have previously reported on patient records kept within the United States Renal Data System (USRDS); ${ }^{9}$ a number of qualitative and quantitative measurements are presented which can be accessed rather easily from the National Institutes of Health. ${ }^{10,11}$ In our previous study, we found that a neural network approach was not superior to that obtainable with a logistic linear approach at predicting time to death. However, since that report, advances in machine learning have allowed for the relatively easy application of other approaches which might help clinicians estimate mortality risk in this population. For that reason, the following study was performed. Methods

Files containing de-identified patient records from the USRDS in 2007 were read in the program SAS (version 9.1), SAS Institute Inc., Cary, NC, and exported in a CSV format. Forty-two variables were selected to be used in the analysis based on their potential clinical significance and their wide availability within the USRDS as we had previously reported. ${ }^{9}$

All analysis was performed using the open source program R. We used a generalized linear model as our default. ${ }^{12}$ In addition, we examined the utility of a support vector machine, 13 decision trees with the RPART package, neural networks (1 hidden layer, feed forward as previously studied(9)), and random forests. ${ }^{14,15}$ The CARET package was used for comparison of the mature models employing $10 \mathrm{~K}$ folds and 3 repeats performed on a training set $(5 \%$ of total) chosen with different randomization seeds to allow for reproducibility. ${ }^{16}$ Other packages within $\mathrm{R}$ were used for different specific tasks (e.g., NNet for construction of the neural network, randomForest (RFor) for constructing random forests) ${ }^{17}$ as we recently demonstrated with the Modification of Diet with Renal Disease (MDRD) dataset. ${ }^{18}$

For these studies, we focused on the categorical 
output of survival less than six months. This outcome variable was chosen for its clinical relevance to nephrology practice. of ischemic heart disease and prevalence of pulmonary disease were both higher in those dying early (both $p<0.01$ ). Many of the data were quite

\section{RESULTS AND DISCUSSION}

In the records that were selected for analysis, just over 67 thousand subjects died within the first six months of starting hemodialysis (HD) therapy whereas the remaining 330 thousand subjects survived longer. The data in these two groups are summarized in Table 1. Those that died early tended to have poorer nutrition as evidenced by a lower serum albumin, serum creatinine $(\mathrm{SCr})$ and body mass index (BMI) (all $p<0.01$ ). They also tended to be significantly older $(68.3+/ 15.0$ vs $61.3+/-$ $15.8, \mathrm{p}<0.01)$, have a lower prevalence of insulin dependent diabetes $(p<0.01)$ and higher EPO dosages $(p<0.01)$. The prevalence

\begin{tabular}{|l|l|l|l|}
\hline & Not Dead at 6 months & Dead at 6 months & $\mathrm{P}$ value \\
\hline $\mathrm{N}$ & 330452 & 67139 & \\
\hline Hemoglobin & $9.79+/-1.67$ & $9.96+/-1.62$ & $\mathrm{P}<0.01$ \\
\hline Albumin & $3.16+/-0.67$ & $2.95+/-0.68$ & $\mathrm{P}<0.01$ \\
\hline SCr & $7.45+/-3.41$ & $6.41+/-3.00$ & $\mathrm{P}<0.01$ \\
\hline BMI & $27.3+/-7.0$ & $26.0+/-6.7$ & $\mathrm{P}<0.01$ \\
\hline BUN & $82.9+/-27.6$ & $83.8+/-29.2$ & $\mathrm{P}<0.01$ \\
\hline SEX & $53 \%$ Male & $53 \%$ Male & $\mathrm{NS}$ \\
\hline RACE & $59 \%$ White 31\% Black & $71 \%$ White $22 \%$ Black & $\mathrm{P}<0.01$ \\
\hline AGE & $10 \%$ other & $7 \%$ other & \\
\hline $\begin{array}{l}\text { DIALYSIS } \\
\text { SETTING }\end{array}$ & $91.3+/-15.8$ & $68.3+/-15.0$ & $\mathrm{P}<0.01$ \\
\hline $\begin{array}{l}\text { DIALYSIS } \\
\text { TYPE }\end{array}$ & $93 \%$ IHD & $93 \%$ In Center & $\mathrm{P}<0.01$ \\
\hline $\begin{array}{l}\text { INCIDENT } \\
\text { ESRD AGE }\end{array}$ & $62.6+/-15.6$ & $96 \%$ IHD & $\mathrm{P}<0.01$ \\
\hline AIDS & $19 \%$ & $69.3+/-14.5$ & $\mathrm{P}<0.01$ \\
\hline HIV & $19 \%$ & $17 \%$ & $\mathrm{P}<0.01$ \\
\hline ALCOH & $1.3 \%$ & $17 \%$ & $\mathrm{P}<0.01$ \\
\hline CANCER & $5.1 \%$ & $2.0 \%$ & $\mathrm{P}<0.01$ \\
\hline CARFAIL & $31 \%$ & $10,2 \%$ & $\mathrm{P}<0.01$ \\
\hline CVA & $9 \%$ & $42 \%$ & $\mathrm{P}<0.01$ \\
\hline INSULIN & $24 \%$ & $13 \%$ & $\mathrm{P}<0.01$ \\
\hline $\begin{array}{l}\text { DIABETES } \\
\text { PRIMARY DX }\end{array}$ & $46 \%$ & $21 \%$ & $\mathrm{P}<0.01$ \\
\hline DRUG & $1.1 \%$ & $41 \%$ & $\mathrm{P}<0.01$ \\
\hline DYYSRYTH & $5.6 \%$ & $1.0 \%$ & $\mathrm{P}<0.05$ \\
\hline EPO & $68 \%$ & $10.4 \%$ & $\mathrm{P}<0.01$ \\
\hline HYPER & $81 \%$ & $75 \%$ & $\mathrm{P}<0.01$ \\
\hline $\begin{array}{l}\text { Ischemic Heart } \\
\text { Disease }\end{array}$ & $24 \%$ & $72 \%$ & $\mathrm{P}<0.01$ \\
\hline MI & $8 \%$ & $\mathrm{P}<0.01$ \\
\hline NOAMBGUL & $3.3 \%$ & $12 \%$ & $\mathrm{P}<0.01$ \\
\hline PERICARD & $0.7 \%$ & $0.1 \%$ & $\mathrm{P}<0.01$ \\
\hline PULMON & $6.9 \%$ & $\mathrm{NS}$ \\
\hline PVASC & $14 \%$ & $4.8 \%$ & $\mathrm{P}<0.01$ \\
\hline SMOKE & $5.6 \%$ & $\mathrm{P}<0.01$ \\
\hline & & $\mathrm{P}<0.01$ \\
\hline
\end{tabular}

TABLE 1: Comparison of Early Death ( $<6$ months) and Others

*Note that because number of subjects is so high in both groups, confidence intervals around point estimate for prevalence are $<<1 \%$ for all categorical values.

$\mathrm{SCr}$ - serum creatinine, BUN - serum urea nitrogen, ALCOH - alcohol dependency, CANCER - cancer present, CARFAIL- cardiac failure, CVA - cerebrovascular accident, HIV - human immunodeficiency virus positive, AIDS - acquired immunodeficiency syndrome present, DRUG - drug dependency, DYYSRYTH- cardiac arrhythmias, EPO - erythropoietin utilization, HYPER - hypertension present, Ischemic Heart Disease present, MI - history of myocardial infarction, NOAMBGUL- not able to ambulate, PERICARD - pericarditis, PULMON - pulmonary disease present, PVASC - peripheral vascular disease present, SMOKE - active smoker. 
similar in the two groups although because of the large numbers involved, statistical significance was noted (Table 1). The high rates of HIV and AIDS reflects the time that these data were obtained; it is quite likely that a more recent data set would have much lower prevalence for HIV and related conditions.

Different machine learning approaches yielded

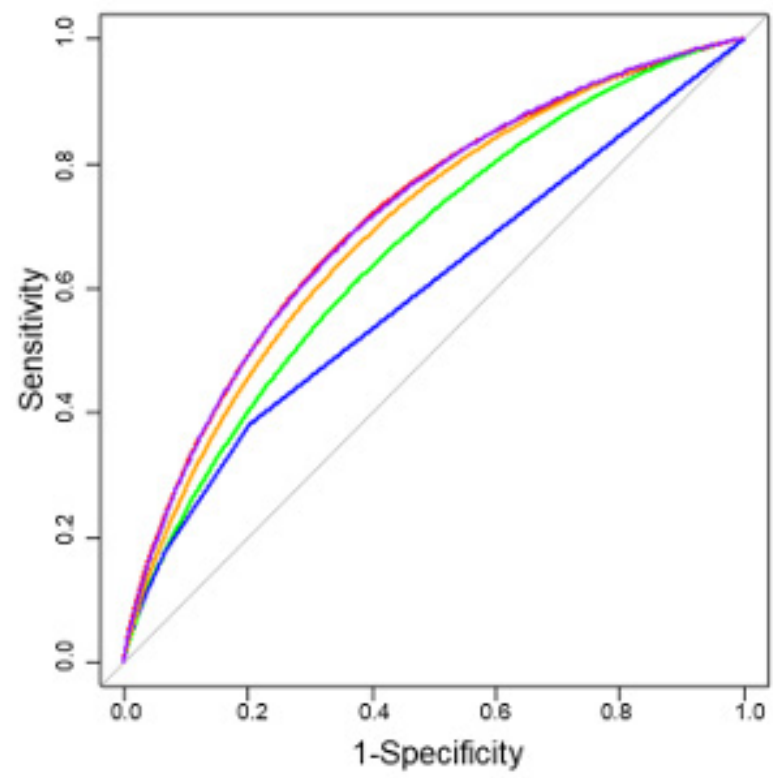

FIGURE 1: Receiver operator curves (ROC) achieved with generalized linear model (GLM) - red, support vector machine (SVM) - green, decision tree (RPart) - blue, feed forward neural network (NNet) - orange and random forest (RFor) - purple on testing set (95\%) after training on training set (5\%) with seed 33 used for randomization.

somewhat different fits as assessed by ROC curves (Figure 1, Table 2). In general, the best fits were obtained by either the generalized linear model (logistic regression, GLM) or the random forest (RForest) approach with the feed-forward neural network (NNet) just slightly behind. The SVM was next with the decision tree (RPart) least effective. Because the decision tree method was so weak, we did not investigate its predictions further. In contrast to the ROC curves which demonstrated significant differences (Table 2), the accuracy values obtained by the remaining four methods were remarkably similar although statistical inferiority to the linear model was evidenced by both the SVM and the NNet models. Accuracy achieved by the Rforest was similar to that obtained by the GLM. Sensitivity of the GLM was inferior to that obtained by the SVM and RForest methods whereas specificity of the NNet method was the best. Along those lines the NNet method had the highest positive predictive value (PPV) where the RForest had the highest negative predictive value (NPV). These data are all summarized in Table 3.

The factors that were most important to the models are shown in Table 4. It is clear that patient age, serum creatinine and serum albumin are important to the different models. Other measurements made it to the top of some of the models but not others. The different models were remarkably consistent with the importance order with which variables were

\begin{tabular}{|l|l|l|l|l|l|}
\hline & GLM & SVM & RPart & NNet & RForest \\
\hline Mean & 0.7140 & 0.6546 & 0.6119 & 0.6980 & 0.7152 \\
\hline SD & 0.0007 & 0.0114 & 0.0087 & 0.0023 & 0.0006 \\
\hline P value & & $\mathrm{P}<0.01$ & $\mathrm{P}<0.01$ & $\mathrm{P}<0.01$ & \\
& & & & & \\
\hline
\end{tabular}
chosen with the different seeds (data not shown).

As the entire data set had a relative paucity of early deaths, we examined how our algorithms performed with a balanced training set

TABLE 2: ROC areas with the different methods: GLM generalized linear model, SVM - support vector machine, RPart - decision tree, NNet - feed forward neural network with 1 hidden layer, RForest - random forest. $P$ value vs GLM. Each ROC determined for each method with 6 different seed values to generate selection of training and testing sets. Training sets chosen to $5 \%$ of the total patient records. constructed from drawing from a subpopulation where the fraction of patients with early $(<6$ month) deaths was 50:50. When we did this, all training algorithms had dramatic increases in kappa values (to about 0.2 ) as well as specificity values (to between 0.60 and 0.65 ) with marked decreases in sensitivity to be essentially matched to the specificity value obtained with that algorithm. As accuracy 


\begin{tabular}{|l|l|l|l|l|l|}
\hline & GLM & SVM & RPart & NNet & RForest \\
\hline Accuracy & $0.8319+/-$ & $0.8311+/-$ & & $0.8272+/-$ & $0.8317+/-$ \\
& 0.0002 & $0.0001^{* *}$ & ND & $0.0004^{* *}$ & 0.0001 \\
\hline Kappa & $0.073+/-$ & $0.013+/-$ & & $0.091+/-$ & $0.048+/-$ \\
& 0.005 & $0.008^{* *}$ & ND & $0.006 * *$ & $0.004 * *$ \\
\hline Sensitivity & $0.989+/-$ & $0.998+/-$ & ND & $0.978+/-$ & $0.993+/-$ \\
& 0.001 & $0.001^{* *}$ & & $0.002^{* *}$ & $0.001^{* *}$ \\
\hline Specificity & $0.058+/-$ & $0.010+/-$ & ND & $0.084+/-$ & $0.037+/-$ \\
& 0.005 & $0.006 * *$ & & $0.006 * *$ & $0.004 * *$ \\
\hline PPV & $0.838+/-$ & $0.832+/-$ & ND & $0.840+/-$ & $0.835+/-$ \\
& 0.001 & $0.001^{* *}$ & & $0.001 * *$ & $0.001^{* *}$ \\
\hline NPV & $0.521+/-$ & $0.491+/-$ & ND & $0.440+/-$ & $0.526+/-$ \\
& 0.009 & $0.032^{* *}$ & & $0.005 * *$ & 0.007 \\
\hline
\end{tabular}

TABLE 3: Diagnostic accuracy with different methods: Calculated from $\mathrm{N}=6$ seeds. Data shown as mean +/- SD of six determinations. PPV - positive predictive value, NPV - negative predictive value. Positive class is "alive $>6$ months." ** $p<0.01$ vs GLM.

\begin{tabular}{|l|l|l|l|l|}
\hline & GLM & SVM & NNet & RForest \\
\hline 1 & Albumin & Age & $\begin{array}{l}\text { Disease } \\
\text { Group }\end{array}$ & Age \\
\hline 2 & Disease Group 81\% & $\begin{array}{l}\text { Incident Age } \\
98 \%\end{array}$ & Age 77\% & $\begin{array}{l}\text { BMI } \\
92 \%\end{array}$ \\
\hline 3 & Non-Ambulatory 78\% & SCr 69\% & SCr 72\% & SCr 86\% \\
\hline 4 & $\begin{array}{l}\text { Hypertension } \\
70 \%\end{array}$ & Albumin 57\% & $\begin{array}{l}\text { Albumin } \\
67 \%\end{array}$ & $\begin{array}{l}\text { BUN } \\
81 \%\end{array}$ \\
\hline 5 & $\begin{array}{l}\text { EPO } \\
58 \%\end{array}$ & DisGrp 45\% & Incident Age 63\% & Albumin 74\% \\
\hline
\end{tabular}

TABLE 4: Variable importance among the different methods Albumin- serum albumin, Incident Age - age of first ESRD treatment, $\mathrm{SCr}$ - serum creatinine, BUN - serum urea nitrogen, EPO - erythropoietin use, Hypertension - presence of hypertension. 
also decreased by about $20 \%$, we chose to leave the training dataset unbalanced. Manuscripts addressing the challenge of unbalanced data sets recognize this problem but do not offer a universal solution. ${ }^{19}$

The results we observed were not very surprising based on our previous experience with this data set where we saw that the neural network model did not afford advantages over linear or actuarial strategies at predicting time to death. 9 In the current study, the logistic linear model (as we were predicting a categorical outcome) was, to all intents and purposes, comparable or superior to more sophisticated strategies at predicting early death after the initiation of dialysis therapy. Cross talk between variables clearly wasn't all that important in the determination of this important outcome; evidence strongly supported the contention that a logistic linear model captured most of the information present in this large data set.

In the analysis performed, sensitivity was calculated based on the model's ability to predict survival. Along with the high prevalence of survivors, the positive predictive value was generally in excess of $80 \%$. This seems to be more than high enough to merit a trial of dialytic therapy. In contrast, the negative predictive value of the models hovered around $50 \%$. Frankly, this does not come close to meeting the authors' threshold for futility of care, and it would seem irresponsible to withhold dialytic therapy for such a prediction. However, it seems that such a prediction might be of a precision sufficient to recommend additional vigilance in monitoring. With the ease of implementing the logistic linear model, this seems to be a reasonable approach based on the data used in this study which are readily available from routine clinical records (and usually submitted with the CMS-2728-U3 form).

\section{AUTHOR AFFILIATIONS}

1. Marshall University Joan C. Edwards School of Medicine, Huntington, West Virginia

2. University of Texas at Southwester, Dallas, Texas

3. University of Toledo, Toledo, Ohio

\section{REFERENCES}

1. Lowrie EG, Lazarus JM, Mocelin AJ, Bailey GL, Hampers $C L$, Wilson $R E$, et al. Survival of patients undergoing chronic hemodialysis and renal transplantation. N Engl J Med. 1973;288(17):8637.

2. Foley RN, Murray AM, Li S, Herzog CA, McBean AM, Eggers PW, et al. Chronic kidney disease and the risk for cardiovascular disease, renal replacement, and death in the United States Medicare population, 1998 to 1999. J Am Soc Nephrol. 2005;16(2):489-95.

3. Port FK, Orzol SM, Held PJ, Wolfe RA. Trends in treatment and survival for hemodialysis patients in the United States. Am J Kidney Dis. 1998;32(6 Suppl 4):S34-8.

4. Block GA, Klassen PS, Lazarus JM, Ofsthun $\mathrm{N}$, Lowrie EG, Chertow GM. Mineral metabolism, mortality, and morbidity in maintenance hemodialysis. J Am Soc Nephrol. 2004;15(8):2208-18.

5. Teng $M$, Wolf $M$, Lowrie $E$, Ofsthun $N$, Lazarus $J M$, Thadhani R. Survival of patients undergoing hemodialysis with paricalcitol or calcitriol therapy. N Engl J Med. 2003;349(5):446-56.

6. Szczech LA, Reddan DN, Klassen PS, Coladonato J, Chua B, Lowrie EG, et al. Interactions between dialysis-related volume exposures, nutritional surrogates and mortality among ESRD patients. Nephrol Dial Transplant. 2003;18(8):1585-91.

7. Lowrie EG, Li Z, Ofsthun N, Lazarus JM. Body size, dialysis dose and death risk relationships among hemodialysis patients. Kidney Int. 2002;62(5):1891-7.

8. Chertow GM, Johansen $\mathrm{KL}$, Lew N, Lazarus $J M$, Lowrie EG. Vintage, nutritional status, and survival in hemodialysis patients. Kidney Int. 2000;57(3):1176-81.

9. Jacob AN, Khuder S, Malhotra N, Sodeman T, Gold JP, Malhotra D, et al. Neural network analysis to predict mortality in end-stage renal disease: application to United States Renal Data System. Nephron Clin Pract. 2010;116(2):c148-58.

10. Description of the USRDS and its data base. Am J Kidney Dis. 1991;18(5 Suppl 2):17-20.

11. USRDS research studies. Am J Kidney Dis. 1991;18(5 Suppl 2):105-10.

12. Gullo CA, McCarthy MJ, Shapiro Jl, Miller BL. Predicting Medical Student Success on Licensure 
Exams. Med Sci Educ. 2015;25:447-53.

13. Tirelli T, Gamba M, Pessani D. Support vector machines to model presence/absence of Alburnus alburnus alborella (Teleostea, Cyprinidae) in North-Western Italy: comparison with other machine learning techniques. C R Biol. 2012;335(10-11):680-6.

14. Chen T, Cao Y, Zhang Y, Liu J, Bao Y, Wang C, et al. Random forest in clinical metabolomics for phenotypic discrimination and biomarker selection. Evid Based Complement Alternat Med. 2013;2013:298183.

15. Khondoker MR, Bachmann TT, Mewissen M, Dickinson P, Dobrzelecki B, Campbell CJ, et al. Multi-factorial analysis of class prediction error: estimating optimal number of biomarkers for various classification rules. J Bioinform Comput Biol. 2010;8(6):945-65.

16. Tsiliki G, Munteanu CR, Seoane JA, FernandezLozano C, Sarimveis H, Willighagen EL. RRegrs: an $R$ package for computer-aided model selection with multiple regression models. J Cheminform. 2015;7:46.

17. Liu R, Li X, Zhang W, Zhou HH. Comparison of Nine Statistical Model Based Warfarin Pharmacogenetic Dosing Algorithms Using the Racially Diverse International Warfarin Pharmacogenetic Consortium Cohort Database. PLoS One. 2015;10(8):e0135784.

18. Khitan Z, Shapiro AP, Shah PT, Sanabria JR, Santhanam P, Sodhi K, Abraham NG, Shapiro J. Predicting adverse outcomes in chronic kidney disease using machine learning methods: data from the modification of diet in renal disease. Marshall Journal of Medicine. 2017;3(4):67-79.

19. Provost F. Machine Learning from imbalanced data sets 101 pages.stern.nyu.edu/ fprovost/ Papers/skew.PDF2000

\section{APPENDIX}

rm(list=Is()) \#empty memory setwd(“C:/Users/shapiroj/Dropbox/Current Stuff/ work") \#set working directory \#load csv file and erase empty columns library(dplyr) dat <- read.csv("esrd.csv",stringsAsFactors=FALSE, na.

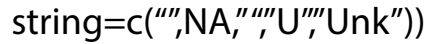

$\operatorname{dim}($ dat $)$ dat1 = dat[,!apply(is.na(dat), 2, all)] \# automatically get rid of empty cols at the end \#set up outcome variable as "yes" or "no" for subsequent machine learning

$\mathrm{A}=\mathrm{NULL}$

$\mathrm{mm}=\operatorname{dim}($ dat1) $[1]$

for(i in $1: \mathrm{mm})\{$

if $($ dat $1[i, 39]<6)\{$

$\mathrm{A}[\mathrm{i}]=$ "yes"

\}else\{

$\mathrm{A}[\mathrm{i}]=$ "no"

\}

\}

\#make all data used for fitting numeric; essential for most machine learning algorithms

dat2 $=$ dat $1[, 1: 38]$

for(i in $1: 38)\{$

dat2[,i]=as.numeric $($ dat2 $[, i])$

\}

\#reconstitute file $z$ with output 1 variable having outcomes as yes or no.

$\mathrm{z}=\mathrm{cbind}($ dat $2, \mathrm{~A})$

colnames(z)[39]="output1"

\#clean up some variables

$\mathrm{z}=\mathrm{z}[,-\mathrm{c}(1,5,38)]$

\#load additional libraries

library(rJava)

library(ROCR)

library(pROC)

library(rpart)

library(caret)

library(nnet)

library(C50)

library(ggplot2)

library(lattice)

library(randomForest)

library(rminer)

library(xgboost)

library(rBayesianOptimization) \#\# Bayesian

Optimization

\#run simulations and save data

$\mathrm{v} v=\mathrm{c}(2,33,15,19,5)$ \#create vector with different seeds

\#loop with different seeds

for(i in 1:5)\{

set.seed(k)

\#split into training and testing subsets based on seed

ind $=$ sample $(2, \operatorname{nrow}(z)$, replace $=$ TRUE, prob $=$ $c(0.5,0.95))$ 
trainset $=z[$ ind $==1$,

testset $=z[$ ind $==2$, ]

\#save files with output data

vvv=paste0("esrd_10_seed_,"k,".txt")

$w w w=p a s t e 0$ ("esrd_10_seed_,"k,.,png")

\#set up training with CARET for different machine

learning methods

control = trainControl (method $=$ "repeatedcv",

number $=10$, repeats $=3$, classProbs $=$

TRUE, summaryFunction = twoClassSummary)

glm.model $=$ train (output $1 \sim$, data $=$ trainset,

method = "glm", metric $=$ "ROC", trControl =

control, preProc $=c($ "center ','scale") $)$

tunGrid_svm $=$ expand.grid(sigma $=c(0.015), C=c(1))$

\#sigma and C fit optimized

separately based on ROC on training set

svm.model $=$ train (output $1 \sim$, data $=$ trainset,

method = "svmRadial",metric $=$ "ROC",

tuneGrid=tunGrid_svm, trControl = control,

preProc $=c($ "center"','scale"))

rpart.model $=$ train (output $1 \sim$. data $=$ trainset,

method $=$ "rpart", metric $=$ "ROC",

trControl = control, preProc $=c($ ("center"','scale"))

tunGrid $=$ expand.grid (size $=c(9)$, decay $=c(0.2))$

\#number of hidden neurons (size) and

decay rate optimized separately based on ROC on

training set

nnet.model $=$ train (output $1 \sim$, data=trainset,

method = "nnet", metric $=$ "ROC",

trace=FALSE, trControl=control, tuneGrid=tunGrid, preProc $=c($ "center','scale"))

tunegrid=expand.grid(.mtry $=c(12))$ \#mtry which is

number of branches to random forest

optimized based on ROC on training set

rfor.model = train (output $1 \sim$., data=trainset,

method = "rf", metric $=$ "ROC",

trControl=control,tuneGrid=tunegrid,

preProc $=c($ "center"','scale"))

\#make predictions based on models

glm.probs = predict(glm.model, testset[,!

names(testset) \%in\% c("output1")], type = "prob")

svm.probs $=$ predict(svm.model, testset[,!

names(testset) \%in\% c("output1")], type = "prob")

rpart.probs = predict(rpart.model, testset[,!

names(testset) \%in\% c("output1")], type = "prob")

nnet.probs=predict(nnet.model, testset[,!

names(testset) \%in\% c("output1")], type = "prob")

rfor.probs=predict(rfor.model, testset[,!

names(testset) \%in\% c("output1")], type = "prob")

\#make ROC graphs png(www)

glm.ROC $=\operatorname{roc}($ response $=$ testset[, c("output1")],

predictor $=$ glm. probs $\$$ yes, levels $=$

levels(testset[, c("output1")]))

$\operatorname{plot}(\mathrm{glm} . \mathrm{ROC}$,add=F, col =" red",main=k)

svm.ROC $=$ roc(response $=$ testset[, c("output1")],

predictor $=$ svm. probs \$yes, levels $=$

levels(testset[, c("output1")]))

plot(svm.ROC, add = TRUE, col ="green")

rpart.ROC $=\operatorname{roc}($ response $=$ testset $[$, c("output 1")],

predictor $=$ rpart.probs \$yes, levels $=$

levels(testset[, c("output1")]))

plot (rpart.ROC, add = TRUE, col ="blue")

nnet.ROC=roc(response $=$ testset[, $c($ "output1")],

predictor $=$ nnet.probs \$yes, levels $=$

levels(testset[, c("output1")]))

plot(nnet.ROC, add = TRUE, col ="orange")

rfor.ROC=roc(response $=$ testset[, c("output1")],

predictor $=$ rfor.probs $\$$ yes, levels $=$

levels(testset[, c("output1")]))

plot(rfor.ROC, add = TRUE, col ="purple")

dev.off() \#close ROC graph

sink(vvv) \#open text output

\#confusion matrices and variable importance lists

glm.pred=predict $(\mathrm{glm}$.

model,testset[!!names(testset)\%in\% c("output1")])

$\mathrm{t}=$ table(glm.pred,testset[,c("output1")])

$\mathrm{tt}=$ confusionMatrix(glm.pred,testset[,c("output1")])

print("glm.model")

print(tt)

$\operatorname{print}(\mathrm{glm} \cdot \mathrm{ROC})$

print(varlmp(glm.model))

svm.pred=predict(svm.

model,testset[,!names(testset)\%in\% c("output1")])

$\mathrm{t}=$ table(svm.pred,testset[,c("output1")])

$\mathrm{tt}=$ confusionMatrix(svm.pred,testset[,c("output1")])

print("svm.model")

print(tt)\#

print(svm.ROC)

print(varlmp(svm.model))

rpart.pred=predict(rpart.

model,testset[!!names(testset)\%in\% c("output1")])

$\mathrm{t}=$ table(rpart.pred,testset[,c("output1")])

$\mathrm{tt}=$ confusionMatrix(rpart.pred,testset[,c("output1")])

print(rpart.ROC)

print(varlmp(rpart.model))

nnet.pred=predict(nnet.

model,testset[!!names(testset)\%in\% c("output1")])

$\mathrm{t}=$ table(nnet.pred,testset[,c("output1")]) 
$\mathrm{tt}=$ confusionMatrix(nnet.pred,testset[,c("output1")])

print("nnet.model")

print(tt)

print(nnet.ROC)

print(varlmp(nnet.model))

rfor.pred=predict(rfor.

model,testset[,!names(testset)\%in\% c("output1")])

$\mathrm{t}=$ table(rfor.pred,testset[,c("output1")])

$\mathrm{tt}=$ confusionMatrix(rfor.pred,testset[,c("output1")])

print("rfor.model")

print(tt)

print(rfor.ROC)

print(varlmp(rfor.model))

sink() \#close text file

\}\#end loop 